\title{
The operation of SEM-EDS analysis in a regulated environment
}

Anthony Hyde ${ }^{1}$, Simon Burgess ${ }^{2}$, Sam Marks $^{2}$, Haithem Mansour ${ }^{2}$ and Richard Mclaughlin ${ }^{3}$

${ }^{1}$ Oxford Instruments, England, United Kingdom, ${ }^{2}$ Oxford Instruments NanoAnalysis, High Wycombe, England, United Kingdom, ${ }^{3}$ Oxford Instruments NanoAnalysis, Concord, United States

This paper looks at the challenges faced by analysts who carry out energy dispersive spectrometry (EDS) in the scanning electron microscope (SEM) in regulated environments, such as those found in pharmaceutical production facilities. The paper will also look at the role of the EDS software in aiding these analysts in conforming to regulations/guidelines such as 'GxP', $21 \mathrm{CFR}$ part 11 and Annex 11.

The FDA have developed two of the most common sets of regulations or guidelines. The first is 21 CFR Part 11, which covers the use of electronic records and signatures in the regulated pharmaceutical, biotechnology and medical device industries. The second is a set of guidelines called GxP, which were created to ensure that products are safe and meet their intended use. 21 CFR Part 11 is intended to ensure that electronic records are trustworthy and reliable. The rules require computer systems and applications to have adequate security features including: audit trails to automatically monitor creation, modification and deletion of electronic records; unique electronic signatures, working backup and recovery with disaster recovery procedures; and effective and rapid archive and restore mechanisms.

Laboratory managers must implement a Standard Operating Procedure for carrying out EDS analysis on samples in the SEM. This SOP will not only help users follow the same process consistently, but will also be used to help prove compliancy when audited. Lab managers look to instrument manufacturers to help simplify their SOPs, by delivering dedicated software that includes; user logins, digital signatures, audit trails and enhanced data security.

For 'Individual User Logins' the solution that best empowers the end-user, is to implement a login system that uses the local windows credentials. This makes it easy for the local IT department to set up and control all aspects of user access to the EDS software. 'Digital Signatures' are a way of electronically tagging all major data interactions within the EDS software such as: user login/image/spectrum/X-Ray map and linescan acquisitions. These digital signatures must contain the username with the date and time. The 'Audit Trail' is the ordered list of digital signatures related to either a project or a user. Users must be able to view records in the audit trail and filter records based on project name, username or date/time. The audit trail cannot be modified or deleted. Users are required to show that their data is secure. This can often be separated into two parts: The first is within the EDS software, where the user must demonstrate that the raw data cannot be deleted or altered. Unlike other techniques, EDS has the benefit that the data in often saved in a propriety file format that cannot be modified. The second is outside the software. Where the data is saved as a 'project' in the users filing system, it must be backed up to a secure server that has restricted access.

Users must also demonstrate the quality of their analysis. They must show an SOP that all users follow. They must also prove that the instrument is performing as designed and delivering data that is in line with the manufacturer's specification. Users will often refer to IQ, OQ and PQ processes to help verify the quality of their data. IQ stands for Installation Qualification and is essentially a proof that an instrument has been installed correctly. OQ is Operational Qualification and PQ is Performance Qualification. For IQ the system must generate an installation report, which states that the system was successfully installed as per the manufacturers guidelines. For OQ and PQ users should have the ability to incorporate energy and beam calibrations into their daily or weekly processes to prove that the system is consistently at the same level of performance. They should also incorporate the measurement of a calibration standard at the start of each day or analysis session to prove the measurement accuracy. Both these events should be recorded in the audit trail. 
Having a simple and straightforward SOP combined with a dedicated EDS software platform is key to carrying out SEM-EDS analysis in a regulated environment. An example workflow for an SOP created using the Oxford Instruments AZtecPharma software, a special version of the AZtecLive software EDS designed for regulated environments, is shown in Fig 1. This new software solution has user logins, digital signatures and an audit trail viewer. In addition, the AZtecPharma software has a separate program called 'Inspector' which is used to view the audit trail - see Fig.2. Therefore, software has an important role in helping users to make their SEM-EDS SOPs not just compliant, but useable.

\section{AZtecPharma Workflow}

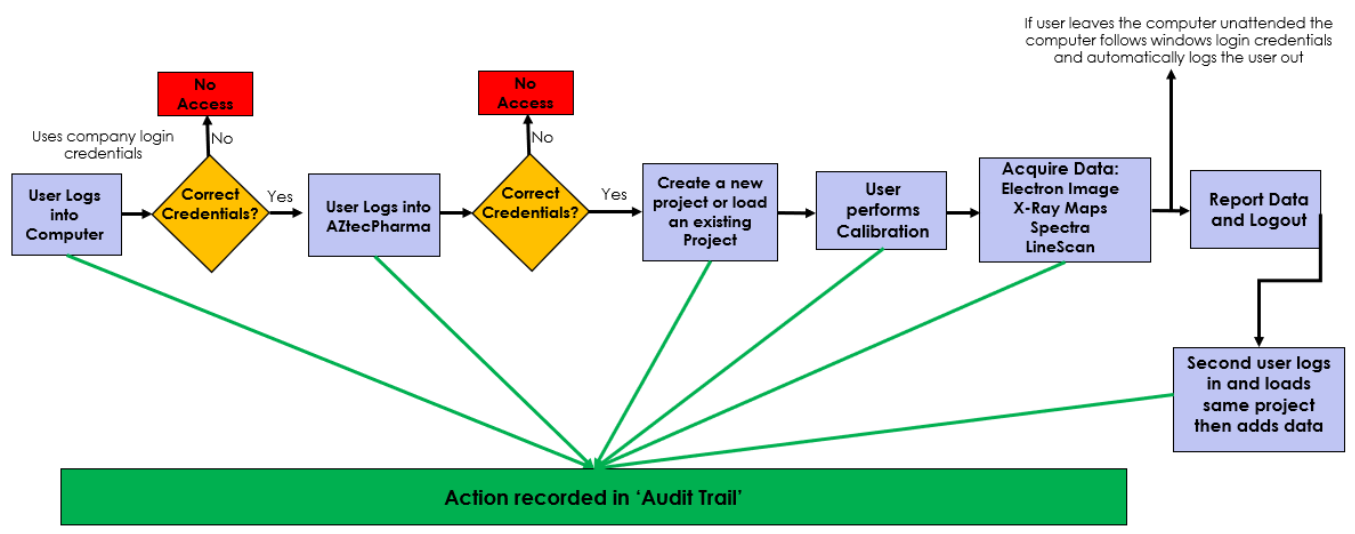

Figure 1. An example workflow for an SOP created using the Oxford Instruments AZtecPharma software

\section{Inspector Software}

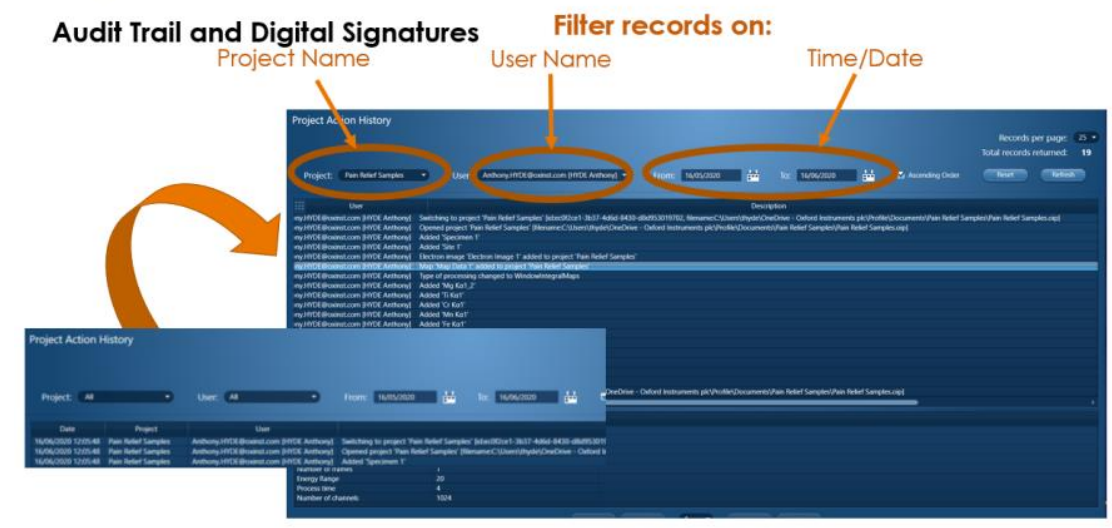

Figure 2. AZtecPharma's audit trail viewer - 'Inspector'

\section{References}

[1] FDA (https://www.fda.gov/downloads/regulatoryinformation/guidances/ucm125125.pdf) 